\title{
Les Hommes XYY
}

\author{
Florence AMBLARD, Pierre JALBERT
}

Laboratoire de Cytogénétique, CHU de Grenoble - PB 317 X, 38043 Grenoble Cedex

\section{RESUME}

La constitution 47,XYY est par ordre de fréquence la 2ème dysgonosomie, elle représente $1 / 851$ à $1 / 1000$ naissances masculines.

Mis à part leur grande taille, ces hommes ne se distinguent en rien de la population générale. Les quelques difficultés d'apprentissage rencontrées sont facilement jugulées par un soutien familial et éducatif, ce d'autant plus facilement qu'elles ne sont pas dues à un retard mental. Une prise en charge adéquate leur permettra de devenir des adultes avec une bonne adaptation familiale, conjugale et sociale. Le plus grand nombre est fertile et leur descendance est normale par élimination au cours de la spermatogenèse de l' Y surnuméraire.

Mots-clés : $47, \mathrm{XYY}$ - grande taille - intelligence normale - fertilité

\section{INTRODUCTION}

SANDBERG et coll. [39] ont les premiers décrit en 1961 la constitution 47,XYY chez un homme d'intelligence normale père de plusieurs enfants. Cependant, de nombreuses enquêtes réalisées par la suite dans l'univers carcéral et les instituts pour délinquants ont cru pouvoir conclure à une tendance naturelle et quasiment irrépressible à l'agressivité des sujets porteurs d'un double $\mathrm{Y}[21,50-51]$.
C'est ainsi que pour les médias, le chromosome Y surnuméraire était devenu "le chromosome du crime", ce qui a évidemment conduit à des excès de toutes sortes. Par exemple une étude danoise [28] réalisée entre 1970 et 1982 montre que la découverte anténatale de cette aneuploïdie est sanctionnée par une interruption médicale de grossesses dans $85 \%$ des cas et seulement dans $79 \%$ des cas lorsqu'il s'agit du syndrome de Klinefelter. L'accumulation de données et le recul du temps permettent d'appréhender avec plus de justesse la réalité des traits en particulier l'intelligence, l'intégration sociale, la fécondité et le devenir de ces hommes.

\section{CYTOGENETIQUE}

\section{Mode de constitution}

Dans les formes homogènes (anomalies présentes dans toutes les cellules), le chromosome Y surnuméraire provient d'une non disjonction lors de la deuxième division méiotique masculine. L'âge paternel ne semble pas la favoriser : les caryotypes des spermatozoïdes des sujets normaux réalisés par fécondation hétérospécifique hommehamster ne montrent pas d'accroissement du nombre de disomie $Y$ avec l'âge [25]. Les techniques d'hybridation in situ utilisant des sondes fluorescentes spécifiques du chromosome Y mettent en évidence deux spots dans 1 à $5 \%$ des spermatozoïdes, ce qui est proche de la fréquence des hommes XYY dans la population générale (14). Le 
léger excès observé (la médiane se trouve à 2,7\%o) pourrait être dû à un biais d'échantillonnage (effectif de l'étude encore restreint) ou au fait que les spermatozoïdes YY serait moins fécondants ce qui reste à prouver. La constitution XYY est la seule anomalie numérique qui ne subit aucune élimination sélective pendant la grossesse [22].

Les formes en mosaïques résultent de non disjonctions mitotiques blastomériques.

\section{Formes Cytogénétiques}

La forme la plus fréquemment rencontrée est la forme homogène $47, \mathrm{XYY}$; plus rarement sont mises en évidence des mosaiques $46, \mathrm{XY} / 47, \mathrm{XYY}$ ou des constitutions à 48 chromosomes : 48, XXYY [35] ou 48, XYYY. Des formes associées à des trisomies autosomiques ont également été décrites [9, 44].

\section{Diagnostic}

Depuis des décennies, deux techniques ont été utilisées pour identifier le ou les chromosomes $\mathrm{Y}$; la plus fiable est la réalisation du caryotype sanguin ; moins onéreuse, mais aussi moins spécifique, est la mise en évidence sur noyau interphasique (frottis sanguin) de l'hétérochromatine de l'Y par des colorations fluorescentes $[30,46]$. Le développement de sondes moléculaires à partir de séquences ADN propres au chromosome $\mathrm{Y}$ permet maintenant un marquage spécifique de ce chromosome après hybridation in situ isotopique ou fluorescente ; ce marquage peut s'opérer aussi bien sur noyaux interphasiques que sur métaphases [14].

\section{FREQUENCE - PHENOTYPE}

La constitution 47,XYY est l'une des aberrations chromosomiques les plus fréquentes. Il était déjà acquis qu'au minimum un garçon sur 1000 en était porteur [10].

Une étude récente [29] réalisée au Danemark sur 34910 nouveaux nés chez lesquels a été pratiqué à titre systématique et consécutif un caryotype sur sang du cordon, à montré une incidence de 1/851 naissances masculines $(1,17 \%)$ plaçant ainsi la constitution 47,XYY au deuxième rang des dysgonosomies après le syndrome de Klinefelter $47, \mathrm{XXY},(1 / 576$ naissances masculines) et juste avant la trisomie de l'X (1/947 naissances féminines). Le Syndrome de Turner est plus rare (1/1893 naissances). Dans cette étude les anomalies chromosomiques touchent 1 enfant sur 276, les anomalies gonosomiques 1 sur 426 , et la trisomie 21,1 sur 592.

Contrairement aux autres anomalies gonosomiques numériques (Turner, Klinefelter) les hommes 47, XYY n'ont pas de phénotype propre en dehors de leur grande taille.

Un certain nombre d'associations malformations ont été publiées mais toutes semblent fortuites ; ce sont des malformations rénales [23] ; osseuses [48] ; génitales (cryptorchidies) [27] ; oculaires (strabisme) [27] ; vertébrales (spina-bifida) [45]. Aucune de ces malformations ne peut être liée à l'état $X Y Y$ puisque leur fréquence n'excède pas dans ce groupe celle de la population générale.

La taille moyenne varie entre 180 et $186 \mathrm{~cm}$ pour des extrêmes à 169 et $223 \mathrm{~cm}$; la fréquence des hommes XYY atteint $10 \%$ chez les sujets de plus de 2 mètres [10]. A la naissance, ces enfants sont de taille normale ; l'accélération de la croissance commence à apparaître vers l'âge de 4 ans [33] et porte surtout sur la longueur des membres inférieurs. Ils atteignent la puberté avec un excès d'environ $7,6 \mathrm{~cm}$; c'est non seulement la vitesse de croissance qui est accélérée mais également la durée de cette croissance ; la taille adulte est atteinte en moyenne 6 mois plus tard (33) (34), le poids est inferieur à celui attendu pour la taille. L'excès de taille à l'âge adulte varie de 9 à $13,1 \mathrm{~cm}$ selon les études. La taille de leurs parents est identique à celle de la population générale. C'est donc bien l'Y surnuméraire qui est en cause bien que les mécanismes cellulaires exacts de cet excès de croissance soient inconnus (hypertestostéronémie ?) [34]. 


\section{DEVELOPPEMENT INTELLECTUEL ET ADAPTATION SOCIALE}

\section{Chez l'enfant}

De nombreuses études prospectives ont été réalisées lorsque le diagnostic est connu dès la naissance par les médecins et les parents. Toutes s'accordent sur le fait qu'il n'existe pas de retard mental chez ces enfants $[10,27,36-37]$. Une étude réalisée dans des instituts médico-pédagogiques n'a d'ailleurs pas mis en évidence d'individus XYY [19].

Une grande disparité des performances semble exister, avec des valeurs extrêmes du quotient intellectuel allant de 77 à 140 [37, 47]. Lorsqu'ils existent, les problèmes psychomoteurs prédominent sur le versant verbal et/ou la lecture. L'élocution et le langage sont alors déficients avec expression difficile et manque de mots ; la compréhension est tout à fait normale [49]. Malgré ces difficultés une faible proportion d'entre eux a recourt à une prise en charge orthophonique. La moitié ont une scolarité normale, l'autre nécessite un soutien scolaire [49] ; $42 \%$ de ces enfants ont des difficultés d'apprentissage de la lecture contre $18 \%$ dans la population contrôle [33].

Lorsqu'on sait les traits particuliers de leur caractère, cet apprentissage un peu chaotique n'a rien d'étonnant. Les études comportementales montrent en effet que ce sont des enfants très distraits, qui ont du mal à focaliser leur attention [49] et à être l'écoute de leur entourage. Ils sont hyperactifs et ne peuvent "tenir en place", bien que réservés, timides et souvent solitaires [33]. Enfants et adolescents ils sont colériques, impulsifs, très émotifs et peu tolérants à l'anxiété [12] mais ne sont pas plus brutaux ou agressifs envers autrui que les enfants du même âge [33]. Leur caractère colérique s'estompe avec l'âge.

NIELSEN [27] a essayé de définir la meilleure scolarité possible pour eux : l'effectif de la classe ne devrait pas être surchargé et l'enseignement devrait prendre en compte le besoin de chacun (ces exigences ne sontelles pas celles de tous les éducateurs et de tous les parents pour leurs enfants ?). Ils doivent être stimulés par leur environnement familial et scolaire ce qui -dit-il-va leur permettre un développement psychomoteur très satisfaisant, identique à celui de la population d'âge égal.

Il est en effet rassurant de constater qu'un environnement familial stable et un soutien lors de l'apparition d'éventuels problèmes d'apprentissage, ne permettent pas de distinguer ces enfants des populations témoins étudiées [36]. Ils suivent une scolarité normale et un certain nombre font des études supérieures brillantes. Ils sont à l'évidence plus fragiles que les autres à un milieu socio-familial défavorable et c'est dans ce cadre que se développent des déviances et des délinquances.

\section{Chez l'adulte}

Les études qui ont conduit à la notion de "chromosome du crime" sont anciennes [18, 21, 50-51]. Des taux élevés d'hommes XYY dans les prisons ont été publiés ; ils sont pour beaucoup liés vraisemblablement à des biais de recrutement et à l'absence de population témoin appariée par la taille. Ainsi une étude réalisée sur 34 individus de grande taille (entre 179 et $210 \mathrm{~cm}$ ) d'une prison australienne [50] trouve une fréquence d'homme XYY de $12 \%$. Lorsqu'on sait que $10 \%$ des hommes de plus de deux mètres sont XYY on s'attend à trouver les mêmes résultats dans une équipe de basket ! Le caryotype de 4139 hommes de grande taille (supérieur au 90ème percentile) a été réalisé au Danemark [51], 12 ont une constitution $47, X Y Y$ soit une incidence de $2,9 \%$, (3 fois la fréquence normale). Les registres pénaux montre que 5 de ces 12 hommes soit $41,7 \%$ ont été condamnés, contre $9,3 \%$ dans la population témoin. Les délits sont la plus part mineurs conduisant à des peines d'emprisonnement de moins d'un an, les actes délictueux n'étant généralement pas dirigés contre autrui. Tous ces individus ont un quotient intellectuel inférieur à la 
moyenne. Ce qui fait dire à WITKIN [51] que l'augmentation de fréquence des hommes XYY dans les prisons n'est pas secondaire à une agressivité exacerbée mais à une intelligence moindre. Peut-être pourrait-on conclure provisoirement que l'on trouve en prison uniquement les hommes XYY les moins intelligents ...

Deux cas d'agressions commises par des sujets XYY viennent d'être récemment publiés [12]. L'un des deux est un malade psychiatrique schizophrène et le second a un lourd passé familial. Comme pour tous les cas de violence de nombreux facteurs contribuent à son expression. La possession de 2 chromosomes $Y$ n'est pas à elle seule une prédisposition au crime.

\section{FERTILITE - DESCENDANCE - SEXUALITE}

\section{Fertilité}

La fertilité des hommes $47, \mathrm{XYY}$ est elle normale ? Il semble aujourd'hui difficile de répondre à cette question avec exactitude : les études sont contradictoires [6-7, 20]. Chandley [7] dans une étude portant sur 1599 sujets stériles trouve que $1,88 \%$ d'entre eux ont deux $\mathrm{Y}$; cette fréquence est très proche de celle observée à la naissance dans la population générale [29]. Au contraire BOURROUILLOU [6] reconnait parmi 952 hommes oligo ou azoospermiques, une fréquence trois fois plus importante $(5,2 \%)$. Il semble donc qu'un certain nombre d'hommes XYY soient normalement fertiles alors que d'autres sont infertiles, les proportions respectives restent à évaluer.

Le caryotype des spermatozoïdes des sujets XYY fertiles réalisés par fécondation hétérospécifique [24] révèle un taux d'anomalie chromosomique identique à celui de la population contrôle $(10,6 \%$ d'anomalies structurales et $4 \%$ d'anomalies numériques) ; tous les spermatozoïdes observés n'ont qu'un seul chromosome sexuel [4]. Les études réalisées à l'aide de sondes moléculaires fluorescentes spécifiques du chromo- some Y présentent les mêmes résultats [15]. Le fait de ne trouver qu'un seul chromosome sexuel dans les spermatozoïdes indique que le chromosome $\mathrm{Y}$ surnuméraire est éliminé durant la spermatogenèse. Il serait perdu aussi bien au cours des mitoses spermatogoniales (selon les sujets 2 à $17 \%$ des spermatogonies possèdent 2 chromosomes $\mathrm{Y}$ ), que lors des divisions méiotiques (moins de $3 \%$ de celles en métaphase de première division ont encore 2 chromosomes $\mathrm{Y}$ ) ; seulement $1 \%$ des spermatozoildes ont 2 Y. Les cellules germinales euploïdes ainsi produites par élimination de l'Y semblent posséder un avantage prolifératif, subissent une méiose normale et aboutissent à la production de gamètes haploïdes.

Ce mécanisme de régulation semble moins efficace chez les sujets XYY oligo ou azoospermes [17]. En réalité la baisse de fertilité serait en rapport avec le moment où l'Y surnuméraire est perdu : plus celui-ci est perdu tard au cours de la spermatogenèse plus le nombre de clones cellulaires $46, \mathrm{XY}$ serait bas [11]. Cela est confirmé par HAN [15]. Chez les sujets qui ont insuffisamment éliminé l'Y surnuméraire, la présence d'un chromosome sexuel non apparié à la méiose pourrait être un facteur de mort cellulaire [43]. On sait que dans la monosomie de l'X, rares sont les ovocytes contenant un seul X arrivant au stade pachytène. La présence d'un univalent $\mathrm{X}$ ou $\mathrm{Y}$ chez les sujets XYY pourrait ainsi aboutir à un arrêt de la maturation germinale. Tout cela reste à l'état d'hypothèse et doit être confirmé, le nombre de sujets étudiés étant encore faible.

\section{La descendance}

La descendance des hommes $47, \mathrm{XYY}$ est habituellement normale ; elle ne présente pas semble-t-il d'excès d'aberrations chromosomiques. Plusieurs publications font pourtant référence à des hommes XYY dont les enfants sont porteurs d'aberrations chromosomiques - trisomie 21 [39], mosaïque $45 \mathrm{X} / 46 \mathrm{XY}$ [45] etc ... Ces études ne révèlent ni l'âge maternel ni l'origine 
paternelle ou maternelle du chromosome surnuméraire. Une revue de la littérature [13] portant sur 57 enfants issus de 31 pères XYY montrait en 1984 que $23 \%$ d'entre eux avaient une anomalie chromosomique ; les aneuploïdies d'origine maternelle ou post zygotique (mosaïques) ne représentaient que $9 \%$ de ces sujets ; cependant un tel excès d'anomalies n'a jamais été confirmé et le caryotype paternel ayant été réalisé après la découverte de l'aberration chromosomique de l'enfant, il est difficile d'admettre à la lumière d'une étude aussi biaisée un excès d'aneuploïdie aussi important [14\%]. Rappelons que les analyses du caryotype des spermatozoildes $[4,15]$ semblent rassurantes puisque l'on ne trouve pas d'accroissement de l'aneuploïdie dans le sperme de ces sujets. Devant des résultats aussi contradictoires, il semble pour l'instant légitime d'une part de ne pas dissuader ces hommes d'avoir une descendance et d'autre part de conseiller par prudence un diagnostic prénatal chromosomique de toutes les grossesses qui en sont issues.

\section{La sexualité}

La constitution XYY prédispose-t-elle à l'homosexualité, à la transexualité, à l'agressivité sexuelle ou à des pratiques déviationnistes?

La littérature se fait l'écho de cas isolés présentant tel ou tel de ces caractères [42]. Il semble en fait que certains de ces hommes ont une activité sexuelle plus importante que les sujets du même âge avec un intérêt déclaré pour des pratiques non conventionnelles [41], mais non violentes ; ils ont pour la plupart un nombre élevé de partenaires féminins [37] ce qui ne les empêche pas d'avoir fréquemment recours à la masturbation. L'importance du désir sexuel a été rattaché, pour certains, à une augmentation de la testostéronémie.

Il est probable qu'à l'image de leur comportement psycho-intellectuel des facteurs biologiques, psychologiques et environnementaux interagissent de manière encore incon- nue et contribuent à l'expression un peu élevée de leur sexualité.

\section{Endocrinologie}

Les testicules des sujets XYY sont de volume normal (20-25 ml), ainsi que la taille de leurs pénis [33]. La structure histologique des gonades est sans particularité chez les sujets fertiles ; les hommes XYY oligospermes ou azoospermes présentent un arrêt de la maturation spermatique à différents stades. Les valeurs des hormones sexuelles sont variables : les concentrations de LH, de FSH et de testostérone sont soit normales, soit augmentées [8,31].

A notre connaissance une seule étude comporte une série témoin [40] ; elle met en évidence un taux de testostérone, de FSH et de LH significativement plus élevés chez les sujets XYY par rapport aux contrôles (appariés par la taille, le quotient intellectuel et le milieu social). Ces écarts n'auraient pu être mis en évidence sans une étude statistique rigoureuse ; la prolactine est normale. Il n'y a pas de corrélation entre les valeurs hormonales et l'hyperactivité sexuelle. On sait que l'administration d'androgène accroît le désir sexuel, un taux endogène d'hormone légèrement augmenté ne semble pas avoir le même effet.

\section{PATHOLOGIE HEMATOLOGIQUE}

Dans les années 70-80, les publications sur les hommes YY avaient comme principal thème le développement psycho-intellectuel. Il est remarquable de constater que depuis une dizaine d'années, la pathologie hématologique a pris le relais. Nombreuses sont les études réalisées qui semblent mettre en évidence une prédisposition des hommes XYY aux leucémies [1-3, 32].

On sait que les anomalies chromosomiques constitutionnelles sont 2 fois plus fréquentes chez les sujets atteints de leucémie que dans la population générale [16,38]. On connaît en particulier depuis fort longtemps l'association entre leucémie aiguë et trisomie 21. Pour certains auteurs [26, 3], l'asso- 
ciation entre la constitution $47, \mathrm{XYY}$ et une fréquence élevée de pathologie maligne ne serait pas fortuite. Sur 1100 hommes porteurs d'hémopathie 5 sont XYY ; cette proportion est presque 5 fois supérieur à celle attendue [1]. Le chromosome $\mathrm{Y}$ jouerait un rôle dans la prolifération cellulaire : les leucémies sont plus fréquentes dans le sexe masculin et la présence d'un Y surnuméraire entraînerait une croissance accrue des tissus qui dérivent du mésoderme (os, dent, tissus hématopoiétiques ...). Le gène d'un facteur de croissance pourrait être localisé sur le chromosome $Y$. Le gène du récepteur de croissance de cellules souches hématopoiétique GM - CSF a bien été localisé sur la région pseudo-autosomique mais rappelons que cette région est commune à l'X et à l'Y [3]. Quoi qu'il en soit il est encore trop tôt pour pouvoir conclure définitivement à une prédisposition des hommes XYY aux leucémies. Des études collaboratives plus étendues sont nécessaires ; elles permettront sans doute de répondre dans un proche avenir.

\section{CONCLUSION}

Le recul du temps et de nombreuses études prospectives permettent maintenant de mieux cerner les hommes $47, \mathrm{XYY}$. Seuls quelques traits de caractères les distinguent des populations témoins. Enfants, ils sont colériques, distraits, fragilisés lorsque le milieu socio familial est instable mais leur développement est satisfaisant lorsqu'ils reçoivent l'aide nécessaire à surmonter les difficultés d'apprentissages inhérentes à leur caractère.

Adultes, ils sont le plus souvent bien insérés dans leur milieu familial et professionnel. Certains, les moins intelligents semblet-il, ou ceux dont l'éducation a été la plus délaissée, ont une conduite délictueuse. Les méfaits commis sont plutôt mineurs bien loin de la conduite fatalement criminelle qui leur était promise naguère. Leur taille est plutôt grande, leur libido élevée, leur fertilité bonne et leur descendance normale.
Il est donc nécessaire et juste, après avoir tant décrié ces sujets, que la société les réhabilite. C'est en particulier le rôle des médecins.

Certaines questions reste posées concernant la méiose de ces sujets et leur prédisposition aux hémopathies.

\section{REFERENCES}

1. ALIMENA G., BILLSTRÖM R., CASALONE R., ET AL. : Cytogenetic Pattern in leukemic cells of patients with constitutional chromosome anomalies. Cancer Genet Cytogenet 1985, 16 : 207-218.

2. ATICHARTAKARN V., PUNYAMMALEE B., WONGSASANT B., ET AL. : Acute nonlymphocytic leukemia with a translocation $(1 ; 3)(\mathrm{p} 36 ; \mathrm{q} 21)$ in a XYY man. Cancer Genet Cytogenet 1986, $21: 79$ 83.

3. BAUDUER F., RAMOND S., DELMER A., ET AL. : Acute Myelomonocytic leukemia in a XYY man.

4. BENET J., MARTIN R.H. : Sperm chromosome complements in a $47, \mathrm{XYY}$ man. Hum Genet 1988 , $78: 313-315$.

5. BENITEZ J., VALCARCEL E., RAMOS C., ET AL. : Frequency of constitutional chromosome alterations in patients with hematologic neoplasias. Cancer Genet Cytogenet 1987, $24: 345$ 354.

6. BOURUOILLOU G, DASTUGUE N, COLOMBIES $P$ : Chromosome studies in 952 infertile males with a sperm count below 10 millions / $\mathrm{ml}$. Hum Genet $1985,71: 366-367$.

7. CHANDLEY A. C., EDMOND P., CHRISTIE S., ET AL : Cytogenetics and fertility in man. Karyotype and seminal analysis. Ann Hum Genet 1975, 39 : 231-254.

8. CHRISTIANSEN P., NIELSEN J. : Urinary follicule hormone and luteinizing hormone in six males with the XYY syndrome. Acta Endocrinologica $1973,73: 625-630$.

9. CROLlA J. A., MACHIN G. A. : 48, XYY, +13 karyotype in a liveborn infant. Clin. Genet. 1980, $17: 31-34$.

10. DE GROUCHY J., TURLEAU C. : Atlas des maladies chromosomiques. Expansion scientifique Française 1982, 400-401.

11. FAED M., ROBERTSON J., MAC INTOSH W. G., ET AL. : Spermatogenesis in an infertile XYY man. Hum Genet 1976, 33 : 341-347.

12. FREYNE A., O' CONNOR A. : XYY genotype and crime : 2 cases. Med Sci Low 1992, $32: 261-263$. 
13. GRASS F., MC COMBS J., SCOTT C. L. ET AL. Reproduction in XYY males : two new cases and implications for genetic counselling. Am. J. Med. Genet. 1984, $19:$ 553-560.

14. GUTTENBACH M., SCHMID M. : Determination of $\mathrm{Y}$ chromosome aneuploïdy in human sperm nuclei by non radioactive in situ hybridization. Am J Hum Genet 1990, 46 : 553-558.

15. HAN T., FORD J. H., FLAHERTY S. P. : A fluorescent in situ hybridization analysis of the chromosome constitution of ejaculated sperm in a $47, \mathrm{XYY}$ male. Clin. Genet. 1994, 45 : 67-70.

16. HECHT F. : Risks of hematologic malignancy with constitutional chromosome abnormalities. Cancer Genet Cytogenet 1987, $24: 375-377$.

17. HUNT P. A., EICHER E. M. : Fertile male mice with three sex chromosomes : Evidence that infertility in XYY male mice is an effect of two Y chromosomes. Chromosoma 1991, $100: 293-299$.

18. HOOK E. B., KIM D. S. : Height and antisocial behaviour in XY and XYY boys. Science 1971, 172 : 284-286.

19.JALBERT P., MALKA C., MOURIQUAND C., ET AL. : Etude des aberrations chromosomiques dans une population de débiles mentaux. Rev. neuropsy. inf. 1972, $20: 761-772$.

20. JALBERT P., SERVOZ-GAVIN M., AMBLARD F., ET AL. : Place du caryotype dans l'exploration de l'infertilité masculine. J. Gynecol. Obstet. Biol. Reprod. 1989, 18 : 724-728.

21. JACOBS P.A., BRUNTON M., MELVILLE M.M., ET AL. : Aggressive behaviour, mental subnormality and the XYY male. Nature, Lond 1965, 208 : 1351.

22. JACOBS P.A. : The role of chromosome abnormalities in reproductive failure. Reprod. Nutr Dev. 1990, suppl $1,63 \mathrm{~S}-74 \mathrm{~S}$.

23. MACHIN C.A. : Urinary tract malformation in the XYY male. Clin. Genet. 1978, 370-372.

24. MARTIN R. H., BALKAN W., BURNS K. ET AL. : The chromosome constitution of 1000 human spermatozoa. Hum. Genet. 1983, $63: 305-309$.

25. MARTIN RH, RADEMAKER AW : The effect of age on the frequency of sperm chromosomal abnormalities in normal men. Am J Genet 1987, 41 : 484-492.

26. MIDRO A. T., WOJTUKIEWICZ M., BIELAWIEC M. ET AL. : XYY syndrome and acute myeloblastic leukemia. Cancer Genet Cytogenet 1987, 24 : 363365 .

27. NIELSEN J., SORENSEN A. M., SORENSEN K. : Follow-up until age 7 to 11 of 25 unselected children. Birth Defects 1982, $18: 61-97$.
28. NIELSEN J., WOHLERT M., FAABERG-ANDERSEN J, ET AL. : Chromosome Examination of 20222 newborn children : results from a 7,5 year study in Aarhus Danemark. Birth Defects 1986, 22 : 209-219.

29. NIELSEN J., WORLERT M. : Chromosome abnormalities found among 34910 newborn children : results from a 13 year incidence study in Aarhus Danemark. Hum Genet 1991, $87: 81-83$.

30. NOEL B. : Identification of 47 , XYY men in the general population by fluorescent heterochromatin. J. Genet. Hum. 1974, $22: 31-43$.

31. PELZMAN K. S., BRODIES H.K.H. : Circulating plasma testosterone in the XYY male. Life sciences $1976,18: 1207-1212$.

32. PEREZ DE OTEYZA J., SUREDA A., FERRO T., ET AL. : Acute lymphoblastic leukemia in an XYY male. Cancer Genet Cytogenet 1990, 39 : 225-227.

33. RATCLIFFE S. G., BUTLER G. E., JONES M. : Edinburgh study of growth and development of children with sex chromosome abnormalities. Birth defects $1990,26: 1-44$.

34. RATCLIFFE S. G., PAN H., MCKIE M. : Growth during puberty in the XYY boy. Ann Hum biol 1992, $19: 579-587$.

35. RINALDI A. : Additional pedigree supporting the origin of XYY from consecutive méiotic non-disjonction in paternal gametogenesis. J. Med. Genet. $1979,16: 225-226$.

36. ROBINSON A., BENDER B. G., BORELLI J., ET AL. : Sex chromosome aneuploidy : prospective and longitudinal studies. Birth defect 1986, $22: 23-43$.

37. ROBINSON A., BENDER B. G., LINDEN M. G. Summary of clinical findings in children young adults with sex chromosome anomalies. Birth defect $1990,26: 225-228$.

38. ROWLEY J. D. : Constitutional chromosome abnormalities in patients with acute nonlymphocytic leukemia. Cancer Genet and Cytogenet 1984, $11: 282$ 283.

39. SANDBERG A. A., KAEPF G. F., ISHIKARCE. : An XYY human male. Lancet ii 1961, 488-489.

40. SCHIAVI R. C., OWEN D., FOGEL M., ET AL : Pituitary gonadal function in XYY and XXY men identified in a population survey. Clin endocrinol 1978, $9: 233-239$.

41. SCHIAVI R. C., THEILGAARD A., OWEN D., ET AL. : Sex chromosome anomalies, hormones and sexuality. Arch Gen psychiatry 1988,45 : 19-21.

42. SNAITH R. P., PENHALE S., HUSFIELD P. : Male to female transsexual with XYY karyotype. Lancet ii 1991, $337: 557-578$. 
43. SPEED R. M., FAED M. J. W., BATSTONE P. J., ET AL. : Persistence of two $Y$ chromosomes through meiotic prophase and metaphase $I$ in an XXY man. Hum Genet 1991, 87 : 416-420.

44. STOLL C., FREY D., WILLARD D. : Down's syndrome with XYY additional aneuploïdy. Hum. Genet. 1976, 34 : 85-88.

45. STOLL C., FLORI E., CLAVERT ET AL. : Abnormal children of a 47 , XYY father. J Med Genet $1979,16: 66-68$.

46. SRIVASTAVA P. K., LUCAS F. V. : Pattern of $Y$ chromatin fluorescence in an XYY man. Clin Genet. 1974, $6: 197-200$.

47. THEILGAARD A. : Psychologic study of XYY and XXY man. Birth defect 1986, $22: 277-292$.

48. THORBUM M.J. : XYY sex chromosoms in a Jamaican with orthopaedic abnomalities. J. Med. Genet. 1968, $5: 3$.

49. WALZER S., BASHIR A. S., SILBERT A. R. : Cognitive and behavioral factors in the learning disabilities of $47, \mathrm{XXY}$ and $47, \mathrm{XYY}$ boys. Birth Defect 1990, 26 : 45-58.

50. WEINER S., SUTHERLAND G., BARTHOLONEW A. : XYY males in a Melbourne prison.Lancet ii 1986, $1: 150$.

51. WITKIN H. A. : Criminality in XYY men. Science $1976,193: 547$.

\section{ABSTRACT XYY Males}

Florence AMBLARD, Pierre JALBERT

Laboratoire de Cytogénétique, CHU de Grenoble, PB 317 X, 38043 Grenoble Cedex
The constitution $47, X Y Y$ is the second most common dysgonosomy, occurring at frequencies of between 1/851 to 1 1.000 male births.

A part from their large size, these men are physically no different from the population in general. Their occasional learning difficulties are easily resolved by family and educational support. As children, they tend to be quick tempered but we not agressive towards others. They have difficulty in concentrating and appear to be disturbed by an unstable family environment which can lead to problems of delinquency. Careful upbringing allows them, as adults to adjust well in family, conjugal and social situations.

The majority are fertile and their offspring normal, since the supernumary $Y$ is eliminated during spermatogenesis. When regulation of this process is defective, oligospermy or azoospermy appears to result. However, the proportion of XYY subjects which are sterile due to this phenomenons is not well known. Likewise, their eventuel predisposition to hematological malignancies has not been well investigated. Further studies should resolve these questions.

Key words : 47,XYY - large size - fertility - normal inteligence 